\title{
Whole exome sequencing with targeted gene analysis and epilepsy after acute symptomatic neonatal seizures
}

\author{
Adam Numis ${ }^{1}$, Gilberto da Gene ${ }^{1}$, Elliott Sherr ${ }^{2}$, and Hannah Glass ${ }^{1}$ \\ ${ }^{1}$ University of California San Francisco \\ ${ }^{2}$ UCSF Benioff Children's Hospital
}

June 15, 2020

\begin{abstract}
The utility of whole exome sequencing (WES) with targeted gene analysis has been important for establishing diagnosis and prognosis of severe early-onset epileptic encephalopathies, but the contribution of pathogenic gene variants to epileptogenesis after acute symptomatic neonatal seizures is not known. We performed a case-control study of 20 trios in children with a history of acute symptomatic neonatal seizures: 10 with and 10 without post-neonatal epilepsy. We used WES and identified pathogenic de novo, transmitted, and non-transmitted variants from genes with known association with epilepsy and correlated prevalence of these variants with epilepsy outcomes. We performed a sensitivity analysis with genes associated with coronary artery disease (CAD). Among 200 known genes associated with epilepsy, we identified pathogenic variants in six children with post-neonatal epilepsy and in two children without subsequent epilepsy (OR 6.0, 95\% CI 0.6-80, p=0.07). There was no difference in the number of children with pathogenic variants in CAD genes between groups. Larger studies evaluating this association may lead to a better understanding of the risk of epilepsy after acute symptomatic neonatal seizures and elucidate molecular pathways that are dysregulated after brain injury and implicated in epileptogenesis.
\end{abstract}

\section{Introduction:}

Neonatal seizures due to brain injury (acute symptomatic seizures) are typically self-limited in the neonatal period, but as many as $25 \%$ of survivors will later develop recurrent unprovoked seizures (epilepsy) and approximately $10 \%$ of survivors are diagnosed with infantile spasms (IS) (Billinghurst et al., 2017; Garfinkle J \& Shevell MI, 2011; Nunes ML, Martins MA, Barea BM, Wainberg RC, \& da Costa JS, 2008; Ronen GM, Buckley D, Penney S, \& Streiner DL, 2007). Known risk factors for epilepsy after acute symptomatic seizures include: severity of neonatal encephalopathy, low birth weight, low blood $\mathrm{pH}$ on the first day of life, abnormal neuroimaging, multifocal (vs. focal) seizures, $>1$ medicine to control neonatal seizures, status epilepticus, persistently abnormal electroencephalogram (EEG) background, and seizure spread to the contralateral hemisphere (Glass, Grinspan, \& Shellhaas, 2018; McDonough et al., 2017; Pisani et al., 2012). Yet, not every neonate with risk factors will develop epilepsy, and the most vulnerable children cannot be prospectively identified. Furthermore, little is known about the pathophysiologic mechanisms of epileptogenesis following neonatal brain injury.

Next-generation sequencing has transformed our understanding of epilepsy genetics; hundreds of genes have been associated with recurrent seizures. Pathogenic variants can result in syndromes with epilepsy as the core symptom (e.g. SCN1A and Dravet syndrome), cause brain malformations and other physical or developmental anomalies associated with epilepsy (e.g. TSC1/2 and tuberous sclerosis complex), or alter inherent seizure susceptibility (e.g. CACNA1H) (Eckle et al., 2014; J. Wang et al., 2017). Whereas the utility of whole exome sequencing (WES) with targeted gene analysis has been important for establishing diagnosis and prognosis of severe early-onset epileptic encephalopathies (Epi25 Collaborative. Electronic address \& 
Epi, 2019; Palmer et al., 2018), the contribution of pathogenic gene variants to epileptogenesis after acute symptomatic neonatal seizures is not known, but we hypothesize that genetic risk factors play a role.

In this pilot case control study, we examined WES in family trios from children affected by acute symptomatic neonatal seizures with and without post-neonatal epilepsy to determine whether there is an increased incidence of de novo and inherited loss of function mutations in known genes associated with epilepsy versus genes in an unrelated group of disorders, in this case those associated with coronary artery disease (CAD). We evaluated remaining WES variants using pathway analysis to evaluate differential enrichment of functional biologic processes in those with and without post-neonatal epilepsy. We hypothesized that 1) pathogenic de novo and inherited mutations in epilepsy-association genes may alter risk of post-neonatal epilepsy after acute symptomatic neonatal seizures, and 2) that there is differential enrichment of pathogenic exome variants in key molecular pathways between those with and without post-neonatal epilepsy.

\section{Methods:}

This was a case-control study of WES family trios including a proband with a history of acute symptomatic seizures and aged at least two years and their biological mother and father. Ten children who developed epilepsy in childhood (cases) were compared with 10 children who remained free from epilepsy until at least 2 years of age (controls). All participants were recruited from the University of California, San Francisco Benioff Children's Hospital (including the Neuro-Intensive Care Nursery follow-up program, Neonatal Seizure Registryresearch study, and Pediatric Epilepsy Center of Excellence) born between 1999 and 2018. We included children with a history of acute symptomatic seizures with onset $<44$ weeks postmenstrual age (Shellhaas et al., 2017). Seizure etiology included, but was not limited to, hypoxic-ischemic encephalopathy (HIE), ischemic stroke, or cerebral hemorrhage (Glass, Numis, Gano, Bali, \& Rogers, 2018). We excluded children with risk for epilepsy independent of seizures and underlying brain injury (including, but not limited, to inborn errors of metabolism or brain malformations), as well as transient cause for seizures (e.g ., mild hypoglycemia, hyponatremia, hypocalcemia with normal neuroimaging), and neonatal-onset epilepsy syndromes. The study protocol was approved by the UCSF Committee on Human Research and both biologic parents of each child provided written informed consent.

Clinical data abstraction: Hospital records were reviewed to determine demographic data, seizure etiology, continuous video EEG results, neuroimaging results, and antiseizure medication use. Neonatal seizure etiology was determined by a pediatric neurologist (ALN and HCG) after reviewing clinical and imaging records and was classified as follows: HIE, ischemic stroke, intracranial hemorrhage, infection, hypoglycemia, or other. Seizure classification (clinical, electroclinical, or electrographic only) and burden in the Neuro-Intensive Care Nursery was determined by a review of the clinical report by a board-certified clinical neurophysiologist (ALN). A neonate was considered to have seizures without EEG confirmation (i.e., clinical seizures) if they had paroxysmal events with a semiology consistent with neonatal seizures warranting treatment with an anti-seizure medication (ASM) before EEG monitoring was initiated. Subclinical, or electrographic only seizures were defined as sudden, abnormal EEG events with repetitive and evolving pattern with amplitude [?]2 $\mu \mathrm{V}$ and duration [?]10 second without a clinical correlate (Tsuchida et al., 2013). Seizure burden was defined as follows: (1) no electrographic seizures, (2) rare electrographic seizures (less than seven), (3) many isolated electrographic seizures (seven or more), (4) frequent recurrent seizures not meeting criteria for status epilepticus, and (5) status epilepticus (Glass et al., 2016).

Outpatient follow-up records from primary care, neurology clinic, subspecialty visits, and the Intensive Care Nursery (ICN) Follow Up Program were reviewed to determine the presence of seizures after the neonatal period. The primary outcome, post-neonatal epilepsy, was defined per International League Against Epilepsy (ILAE) 2014 criteria (Fisher et al., 2014). IS was defined according to ILAE criteria as seizures characterized by "epileptic spasms... a sudden flexion, extension, or mixed extension-flexion of predominantly proximal and truncal muscles" occurring in clusters and during infancy. Intractable epilepsy was defined as failure of two appropriate ASMs.

Genetic sampling, whole exome capture and sequencing: After consenting to participate in this study, families 
were mailed validated self-collection and assisted collection saliva-based collection kits (DNA Genotek OGR500 and OGR-575). Samples was returned and stored at $4^{\circ} \mathrm{C}$ until processing at the UCSF Institute for Human Genetics Genomics Core. DNA was isolated using the Qiagen Gentra Puregene system. DNA was fragmented using a Covaris LE220 to a size range of $\sim 350$ bases and assembled into a library constructed with unique dual indexes compatible with NovaSeq. As previously described, exome sequencing was performed using the NimbleGen Human SeqCap EZ Exome (v3.0) kit according to the manufacturer's protocol.(Marco et al., 2018) Libraries were pooled into a capture reaction that contains biotinylated oligonucleotide probes to target specific regions of interest. The biotinylated probe/target hybrids were pulled down by streptavidincoated magnetic beads to obtain libraries highly enriched for the target regions. WES was performed using the Illumina NovaSeq 6000. Sequencing data were transferred using gzipped fastq format for analysis.

Exome Data Analysis : In our primary analysis, we restricted WES data to 200 genes found in commercially available epilepsy gene panels (GeneDx "Comprehensive epilepsy panel", Gaithersburg, MD; and, Invitae "Epilepsy Panel", San Francisco, CA; Supplemental Table 1). As a sensitivity analysis, we restricted WES data to a subset of 89 non-overlapping genes associated with CAD as previously described (Marco et al., 2018). In a secondary analysis we analyzed the complete WES data set.

Our analytic pipeline followed 'The Broad Institute's Best Practices' guidelines for discovering putative variants and utilizes the Genome Analysis Toolkit (GATK; software version 2014.23.1.7-10) in combination with BWA-mem, Picard Tools, and SAM Tools as previously described ("Genome Analysis Toolkit. Broad Institute, MIT/Harvard (MA)."). In brief, after aligning the DNA read sequences to the GRCh37 reference build using BWA-mem, Picard Tools were used to identify and remove PCR duplicates, add read group information, and sort alignment files using modules Mark Duplicates, SortSam, and AddOrReplaceReadGroups respectively. All variants were compared to parental samples to determine if they were de novo or inherited from the biological mother or father.

For all analyses, variants were required to be within the transcript region (identified as a missense/nonsense single nucleotide variant or out of frame small insertion or deletion (indel)) or within 3 base pairs of a splice site, be below a population frequency of $0.1 \%$ (as determined by 1000 Genomes and the Exome Variant Server 6500), a CADD score of greater than 20, and genotype quality (GQ) of greater than 50. For de novo analyses all variants had a minimum of 10 reads with at least 3 showing the alternate variant in addition to an allelic balance greater than 0.25 . In targeted gene sets allelic balance requirement was lowered to 0.1 . In both analyses, parents were required to have a minimum GQ of 50 with no reads showing the alternate variant. For inheritance analysis in targeted gene panels, variants were separated into sub-groups of transmitted (passed from parent to child) or non-transmitted (not passed from parent to child).

Variant Classification : Each variant was annotated against a reference transcript. In silico modeling with Polyphen-2 (HumDiv and HumVar) was used to assess protein structure/function and evolutionary conservation. Variants were classified as 'pathogenic', 'benign', or a 'variant of uncertain significance' (VUS), according to the American College of Medical Genetics and Genomics (ACMG) guidelines (Richards et al., 2015). In brief, de novo variants in the proband were classified as pathogenic if a null variant (nonsense, frameshift, canonical +/- 1 or 2 splice sites) OR Polyphen-2 predicted a deleterious effect on the gene product. Inherited variants were classified as pathogenic if a null variant OR Polyphen-2 predicted a deleterious effect on the gene product and either: the variant was previously observed in unrelated patients with epilepsy OR the variant was absent from controls in population databases and located in well-established functional domain without benign variation. Variants were classified as benign when a single variant was identified in a gene associated with an autosomal recessive disorder (carrier state) OR met two of three following criteria: 1) Polyphen-2 modeling predicted no effect on the gene product; 2 ) the variant was previously reported as benign; 3) the variant was observed in a healthy adult in a disorder with full penetrance expected at an early age. Variants were classified as VUS when not meeting criteria for a pathogenic or benign variant. The biological relevance of all affected variants was evaluated using the Online Mendelian Inheritance in Man database (OMIM), ClinVar, gnomAD, and Uniprot (Karczewski et al., 2020; Landrum et al., 2018; "Online Mendelian Inheritance in Man, OMIM@. McKusick-Nathans Institute of Genetic Medicine, Johns Hopkins 
University (Baltimore, MD)," ; UniProt, 2019).

KEGG pathway enrichment analysis : Pathogenic de novovariants identified in the complete WES dataset were analyzed for functional properties using Kyoto Encyclopedia of Genes and Genomes (KEGG) searches (Kanehisa, 2019; Kanehisa \& Goto, 2000; Kanehisa, Sato, Furumichi, Morishima, \& Tanabe, 2019). Given the limitations of power with sample size, we limited pathway analysis to KEGG orthology and excluded categorization of human disease (09160) and organismal systems (09150) apart from the nervous system (09156).

Statistical analyses : Statistical analyses were performed using Stata 15.1 software. Chi-square test was used to compare categorical variables and $t$ test for continuous variables. Significance was determined as p $<0.05$.

\section{Results:}

We conducted WES in 20 trios, of whom 10 probands developed post-neonatal epilepsy at a median age of 16 months (IQR 5-24 months). Median age of follow-up in children without epilepsy was 3.2 years (IQR 2.5-3.8), with $70 \%$ of children having more than 3 years of follow-up and no child having more than 5 years of follow-up.

In children with post-neonatal epilepsy, five were diagnosed with IS, of whom two had HIE, one had ischemic stroke, one had intracranial hemorrhage, and one had infection as cause of their acute symptomatic neonatal seizures. Children with and without post-neonatal epilepsy did not differ by sex, mode of delivery, gestational age, birth weight, neonatal seizure burden, or seizure treatment in the neonatal period (Table 1). The underlying etiologies for neonatal seizures were similar between groups, with HIE, ischemic stroke and intracranial hemorrhage accounting for the majority in children with and without post-neonatal epilepsy.

Targeted Gene Analysis (Epilepsy Gene Panel) Analysis : Among 200 genes associated with epilepsy, we identified 29 variants: 4 de novo variants in 3 genes and 25 inherited variants in 23 genes. Nine (31\%) of the 29 variants were classified as pathogenic (Table 2), 16 (55\%) as benign, and the remaining 4 (14\%) as VUS (Supplemental Table 2). There was no difference in variant type (missense, nonsense, frameshift, splice site) between children with and without post-neonatal epilepsy.

The nine pathogenic variants in epilepsy-associated genes were more common among children with postneonatal epilepsy (6/10 children, $60 \%)$ as compared to those without $(2 / 10,20 \%)$. Children with epilepsy had 6.0 times the odds of having a pathogenic variant compared to those without post-neonatal epilepsy ( $95 \%$ confidence interval $0.6-80, \mathrm{p}=0.07)$. In contrast, in a similar analysis using known CAD genes, two children with post-neonatal epilepsy and two children without epilepsy had pathogenic variants identified (OR: 1.0, 95\% CI: 0.06-17, $\mathrm{p}=1.0)$. Similarly, there was no difference in the number of families with non-transmitted pathogenic variants in epilepsy or CAD genes (in each analysis, two parents of children with epilepsy and one parent of a child without epilepsy had pathogenic variants identified, OR: 2.25, 95\% CI: 0.1-147, $\mathrm{p}=0.53$ ).

Among children with post-neonatal epilepsy, four of the seven pathogenic variants were in genes that have been associated with altered susceptibility to epilepsy (CACNA1H, CASK, RBFOX3 and RYR3 ) (Eckle et al., 2014; Euro, Epilepsy Phenome/Genome, \& Epi, 2014; Lal et al., 2013; Srivastava et al., 2016), and three were in genes that are associated with epilepsy syndromes with incomplete penetrance and variable expressivity (KCNT1 , MAGI2, andPRRT2 ) (Moller et al., 2015; Rothlisberger, Hoigne, Huber, Brunschwiler, \& Capone Mori, 2010; van Vliet et al., 2012). Four of five children with IS had pathogenic variants. Among the children without post-neonatal epilepsy, the two pathogenic variants identified were in genes that can result in epilepsy with onset through 12-18 years of age, outside the window of follow-up in this cohort ( $C P A 6$ and $P O L G$ ) (Anagnostou, Ng, Taylor, \& McFarland, 2016; Rothlisberger et al., 2010; Salzmann et al., 2012; Sapio et al., 2015).

WES Analysis with KEGG Orthology: Seventeen pathogenic de novo variants were found in genes without a known association with epilepsy (Table 3). Seven pathogenic variants were found in six children with post-neonatal epilepsy and 10 pathogenic variants were found in six children without post-neonatal epilepsy (OR 1.0, 95\% CI 0.11-8.4, $\mathrm{p}=1.0$ ). There was no difference between groups with respect to variant type 
(missense, nonsense, frameshift, splice site). KEGG orthology analysis demonstrated children who developed post-neonatal epilepsy had a relative enrichment in variants associated with the nervous system, including synaptic transmission and those without epilepsy had a relative enrichment of variants associated with cell growth and death, in particular the ubiquitin system (Figure 1).

\section{Discussion:}

In this pilot case-control study of 20 trios of children with a history of acute symptomatic seizures with and without subsequent epilepsy, WES with targeted analysis of epilepsy associated genes identified 9de novo or inherited pathogenic variants in 10 children. Children with epilepsy had increased odds of having a pathogenic variant compared to those without post-neonatal epilepsy. There was no difference in the odds of having a pathogenic variant in CAD genes, or a difference in the odds non-transmitted variants in epilepsy or CAD genes between groups, suggesting that the findings are related to the development of epilepsy. Our findings add to the growing literature about the use of genetic testing to understand epilepsy. Targeted gene panels and WES are considered important for defining diagnosis and understanding prognosis of for a wide range of non-acquired epilepsies from severe early-onset epileptic encephalopathies to focal epilepsies in adulthood (Epi25 Collaborative. Electronic address \& Epi, 2019; Epi \& Epilepsy Phenome/Genome, 2017; Palmer et al., 2018). If replicated in a larger cohort, our findings suggest that genetic testing may also play a role in understanding epilepsy after acute neonatal symptomatic seizures

Identification of pathogenic variants in genes associated with epilepsy may also inform anti-seizure medication (ASM) management. For example, in our cohort, two children with intractable post-neonatal epilepsy had variants in the $C A C N A 1 H$ and $C A S K$ genes. The $C A C N A 1 H$ gene encodes a subunit of the voltagedependent calcium channel complex. CACNA1H-associated epilepsy has demonstrated responsiveness to lamotrigine or ethosuximide therapy (Eckle et al., 2014; Glauser et al., 2017). The $C A S K$ gene encodes the protein calcium/calmodulin-dependent serine protein kinase regulating alpha-amino-3-hydroxy-5-methyl-4isoxazolepropionic acid (AMPA) receptor trafficking (Gao et al., 2019; Lin, Jeyifous, \& Green, 2013). Perampanel is a selective, non-competitive AMPA agonist and with potential to rescue CASK-mediated disruption. In our cohort, children with pathogenic variants in these genes have at least weekly seizures, three or more ASMs have failed to control the seizures, and had not yet trialed possible precision medicine therapies. Targeted gene analysis may inform providers regarding ASM selection, providing an individualized approach to epilepsy management.

WES with gene set enrichment analysis to compile an individual's genetic variant burden in pathways that are over- or under-represented can inform exploration of molecular processes that may facilitate, or suppress epileptogenesis (Bakir-Gungor, Baykan, Ugur Iseri, Tuncer, \& Sezerman, 2013; He, Xiao, \& Lv, 2014). In our secondary analysis, WES analysis demonstrated a similar number of de novovariants throughout the exome among those with and without epilepsy after acute symptomatic neonatal seizures. KEGG orthology analysis revealed differences in the relative enrichment of variants in key molecular processes between groups. Notably, those with post-neonatal epilepsy had enrichment of pathogenic variants associated with synaptic transmission while those without post-neonatal epilepsy had enrichment of pathogenic variants in cell growth and death pathways, in particular the ubiquitin pathway. This finding is compelling, given that epilepsy is caused by an imbalance between neuronal excitation and inhibition and alterations in synaptic transmission contribute to the disease process, although the impact of the pathogenic variants in our cohort is not known. Ubiquitin is a regulatory protein associated with neurologic disease through its effects on neural development and maintenance via post-translational modifications and resultant protein degradation (Zheng et al., 2016). Levels of the brain enriched enzyme ubiquitin C-terminal hydrolase-L1 (UCH-L1) can predict neuronal injury after traumatic brain injury, ischemic brain injury and neonatal HIE (Massaro et al., 2018; Wang, Yang, Sarkis, Torres, \& Raghavan, 2017). These data can inform future evaluation of single nucleotide polymorphisms (SNPs) that alter function within these pathways with the aim of improving our understanding mechanism of epileptogenesis after brain injury (Diamond et al., 2014).

This single-center study has limitations. First, the small sample size limits immediate generalizability. Second, although we only studied children whose epilepsy onset was before age two years, the duration follow-up 
for children without epilepsy was relatively short (two to five years), and so these children may yet develop epilepsy (Fox, Glass, Sidney, Smith, \& Fullerton, 2016; Pisani et al., 2018). For example, the pathogenic variants in the CPA6 and POLG genes identified in our control group (children without epilepsy through at least 2 years of age) increase epilepsy susceptibility into late childhood; longer duration of follow-up could result in re-classification of these probands (Gao et al., 2019; Glauser et al., 2017; Lin et al., 2013). Third, gene sequencing has inherent limitations in predicting the consequences of DNA variants on protein function. Our methods use ACMG criteria for variant classification so as to use best practices and limit future re-categorization (Richards et al., 2015).

If the role of targeted gene analysis to predict post-neonatal epilepsy after acute symptomatic seizures is supported with future studies, epilepsy gene panels could enhance established prediction paradigms that currently incorporate clinical, EEG, and radiologic data, allowing for improved counseling of providers and families (Fox et al., 2016; Glass, Grinspan, et al., 2018; Glass, Numis, et al., 2018; McDonough et al., 2017; Pisani et al., 2018). Future investigations of genetic sequencing in larger cohorts, perhaps leveraging existing databases of neonates with acute symptomatic seizures with longer follow-up duration will allow for improved estimates of test sensitivity and specificity as well as multivariate modeling with known risk factors of post-neonatal epilepsy. WES in larger cohorts also will allow robust bioinformatic analysis and hierarchical clustering to visualize and explore functional pathways associated with epilepsy after acute symptomatic seizures (Lee et al., 2016; Lee et al., 2019).

\section{Acknowledgements:}

This study was supported by a Marcus Program Seeding Bold Ideas Award. The authors thank Renée Shellhaas, MD for her review of the manuscript and Rebecka Craig for her contributions to the investigation.

Conflicts of Interest: The authors report no potential sources of conflict of interest.

Data Availability: The data that support the findings of this study are available on request from the corresponding author. The data are not publicly available due to privacy or ethical restrictions.

\section{References}

Anagnostou, M. E., Ng, Y. S., Taylor, R. W., \& McFarland, R. (2016). Epilepsy due to mutations in the mitochondrial polymerase gamma (POLG) gene: A clinical and molecular genetic review. Epilepsia, 57 (10), 1531-1545. doi:10.1111/epi.13508

Bakir-Gungor, B., Baykan, B., Ugur Iseri, S., Tuncer, F. N., \& Sezerman, O. U. (2013). Identifying SNP targeted pathways in partial epilepsies with genome-wide association study data. Epilepsy Res, 105 (1-2), 92-102. doi:10.1016/j.eplepsyres.2013.02.008

Billinghurst, L. L., Beslow, L. A., Abend, N. S., Uohara, M., Jastrzab, L., Licht, D. J., \& Ichord, R. N. (2017). Incidence and predictors of epilepsy after pediatric arterial ischemic stroke. Neurology, 88 (7), 630637. doi:10.1212/WNL.0000000000003603

Diamond, M. L., Ritter, A. C., Failla, M. D., Boles, J. A., Conley, Y. P., Kochanek, P. M., \& Wagner, A. K. (2014). IL-1beta associations with posttraumatic epilepsy development: a genetics and biomarker cohort study. Epilepsia, 55 (7), 1109-1119. doi:10.1111/epi.12628

Eckle, V. S., Shcheglovitov, A., Vitko, I., Dey, D., Yap, C. C., Winckler, B., \& Perez-Reyes, E. (2014). Mechanisms by which a CACNA1H mutation in epilepsy patients increases seizure susceptibility. $J$ Physiol, 592 (4), 795-809. doi:10.1113/jphysiol.2013.264176

Epi25 Collaborative. Electronic address, s. b. u. e. a., \& Epi, C. (2019). Ultra-Rare Genetic Variation in the Epilepsies: A Whole-Exome Sequencing Study of 17,606 Individuals.Am J Hum Genet, 105 (2), 267-282. doi:10.1016/j.ajhg.2019.05.020

Epi, K. c., \& Epilepsy Phenome/Genome, P. (2017). Ultra-rare genetic variation in common epilepsies: a case-control sequencing study. Lancet Neurol, 16 (2), 135-143. doi:10.1016/S1474-4422(16)30359-3 
Euro, E.-R. E. S. C., Epilepsy Phenome/Genome, P., \& Epi, K. C. (2014). De novo mutations in synaptic transmission genes including DNM1 cause epileptic encephalopathies.Am J Hum Genet, 95 (4), 360-370. doi:10.1016/j.ajhg.2014.08.013

Fisher, R. S., Acevedo, C., Arzimanoglou, A., Bogacz, A., Cross, J. H., Elger, C. E., . . . Wiebe, S. (2014). ILAE official report: a practical clinical definition of epilepsy. Epilepsia, 55 (4), 475-482. doi:10.1111/epi.12550

Fox, C. K., Glass, H. C., Sidney, S., Smith, S. E., \& Fullerton, H. J. (2016). Neonatal seizures triple the risk of a remote seizure after perinatal ischemic stroke.Neurology, 86 (23), 2179-2186. doi:10.1212/WNL.0000000000002739

Gao, R., Zaccard, C. R., Shapiro, L. P., Dionisio, L. E., Martin-de-Saavedra, M. D., Piguel, N. H., . . . Penzes, P. (2019). The CNTNAP2-CASK complex modulates GluA1 subcellular distribution in interneurons. Neurosci Lett, 701 , 92-99. doi:10.1016/j.neulet.2019.02.025

Garfinkle J, \& Shevell MI. (2011). Cerebral palsy, developmental delay, and epilepsy after neonatal seizures. Pediatric Neurology, $44,88-96$.

Genome Analysis Toolkit. Broad Institute, MIT/Harvard (MA). Retrieved from https://software.broadinstitute.org/gatk/best-practices/bp_3step.php?case=GermShortWGS

Glass, H. C., Grinspan, Z. M., \& Shellhaas, R. A. (2018). Outcomes after acute symptomatic seizures in neonates. Semin Fetal Neonatal Med, 23 (3), 218-222. doi:10.1016/j.siny.2018.02.001

Glass, H. C., Numis, A. L., Gano, D., Bali, V., \& Rogers, E. E. (2018). Outcomes After Acute Symptomatic Seizures in Children Admitted to a Neonatal Neurocritical Care Service.Pediatr Neurol, 84, 39-45. doi:10.1016/j.pediatrneurol.2018.03.016

Glass, H. C., Shellhaas, R. A., Wusthoff, C. J., Chang, T., Abend, N. S., Chu, C. J., . . Neonatal Seizure Registry Study, G. (2016). Contemporary Profile of Seizures in Neonates: A Prospective Cohort Study. $J$ Pediatr, 174, 98-103 e101. doi:10.1016/j.jpeds.2016.03.035

Glauser, T. A., Holland, K., O'Brien, V. P., Keddache, M., Martin, L. J., Clark, P. O., . . . Childhood Absence Epilepsy Study, G. (2017). Pharmacogenetics of antiepileptic drug efficacy in childhood absence epilepsy. Ann Neurol, 81 (3), 444-453. doi:10.1002/ana.24886

He, K., Xiao, W., \& Lv, W. (2014). Comprehensive identification of essential pathways and transcription factors related to epilepsy by gene set enrichment analysis on microarray datasets. Int J Mol Med, 34 (3), 715-724. doi:10.3892/ijmm.2014.1843

Kanehisa, M. (2019). Toward understanding the origin and evolution of cellular organisms.Protein Sci, 28 (11), 1947-1951. doi:10.1002/pro.3715

Kanehisa, M., \& Goto, S. (2000). KEGG: kyoto encyclopedia of genes and genomes. Nucleic Acids Res, 28 (1), 27-30. doi:10.1093/nar/28.1.27

Kanehisa, M., Sato, Y., Furumichi, M., Morishima, K., \& Tanabe, M. (2019). New approach for understanding genome variations in KEGG. Nucleic Acids Res, 47 (D1), D590-D595. doi:10.1093/nar/gky962

Karczewski, K. J., Francioli, L. C., Tiao, G., Cummings, B. B., Alföldi, J., Wang, Q., . . Genome Aggregation Database, C. (2020). The mutational constraint spectrum quantified from variation in 141,456 humans. Nature, 581 (7809), 434-443. doi:10.1038/s41586-020-2308-7

Lal, D., Reinthaler, E. M., Altmuller, J., Toliat, M. R., Thiele, H., Nurnberg, P., . . . Neubauer, B. A. (2013). RBFOX1 and RBFOX3 mutations in rolandic epilepsy.PLoS One, 8 (9), e73323. doi:10.1371/journal.pone.0073323 
Landrum, M. J., Lee, J. M., Benson, M., Brown, G. R., Chao, C., Chitipiralla, S., . . Maglott, D. R. (2018). ClinVar: improving access to variant interpretations and supporting evidence. Nucleic Acids Res, 46 (D1), D1062-D1067. doi:10.1093/nar/gkx1153

Lee, S., Choi, S., Kim, Y. J., Kim, B. J., Consortium, T. d.-G., Hwang, H., \& Park, T. (2016). Pathwaybased approach using hierarchical components of collapsed rare variants. Bioinformatics, 32 (17), i586-i594. doi:10.1093/bioinformatics/btw425

Lee, S., Kim, S., Kim, Y., Oh, B., Hwang, H., \& Park, T. (2019). Pathway analysis of rare variants for the clustered phenotypes by using hierarchical structured components analysis. BMC Med Genomics, 12 (Suppl 5), 100. doi:10.1186/s12920-019-0517-4

Lin, E. I., Jeyifous, O., \& Green, W. N. (2013). CASK regulates SAP97 conformation and its interactions with AMPA and NMDA receptors. J Neurosci, 33 (29), 12067-12076. doi:10.1523/JNEUROSCI.0816-13.2013

Marco, E. J., Aitken, A. B., Nair, V. P., da Gente, G., Gerdes, M. R., Bologlu, L., . . Sherr, E. H. (2018). Burden of de novo mutations and inherited rare single nucleotide variants in children with sensory processing dysfunction. BMC Med Genomics, 11 (1), 50. doi:10.1186/s12920-018-0362-x

Massaro, A. N., Wu, Y. W., Bammler, T. K., Comstock, B., Mathur, A., McKinstry, R. C., . . Juul, S. (2018). Plasma Biomarkers of Brain Injury in Neonatal Hypoxic-Ischemic Encephalopathy. J Pediatr, 194, 67-75 e61. doi:10.1016/j.jpeds.2017.10.060

McDonough, T. L., Paolicchi, J. M., Heier, L. A., Das, N., Engel, M., Perlman, J. M., \& Grinspan, Z. M. (2017). Prediction of Future Epilepsy in Neonates With Hypoxic-Ischemic Encephalopathy Who Received Selective Head Cooling. J Child Neurol, 32 (7), 630-637. doi:10.1177/0883073817698628

Moller, R. S., Heron, S. E., Larsen, L. H., Lim, C. X., Ricos, M. G., Bayly, M. A., . . Dibbens, L. M. (2015). Mutations in KCNT1 cause a spectrum of focal epilepsies.Epilepsia, 56 (9), e114-120. doi:10.1111/epi.13071

Nunes ML, Martins MA, Barea BM, Wainberg RC, \& da Costa JS. (2008). Neurological outcomes of newborns with neonatal seizures. Arq Neuropsiquiatr, 66 , 168-174.

Online Mendelian Inheritance in Man, OMIMß. McKusick-Nathans Institute of Genetic Medicine, Johns Hopkins University (Baltimore, MD). (Mar 2020). Retrieved from https://omim.org/

Palmer, E. E., Schofield, D., Shrestha, R., Kandula, T., Macintosh, R., Lawson, J. A., . . Sachdev, R. K. (2018). Integrating exome sequencing into a diagnostic pathway for epileptic encephalopathy: Evidence of clinical utility and cost effectiveness. Mol Genet Genomic Med, 6 (2), 186-199. doi:10.1002/mgg3.355

Pisani, F., Facini, C., Bianchi, E., Giussani, G., Piccolo, B., \& Beghi, E. (2018). Incidence of neonatal seizures, perinatal risk factors for epilepsy and mortality after neonatal seizures in the province of Parma, Italy. Epilepsia, 59 (9), 1764-1773. doi:10.1111/epi.14537

Pisani, F., Piccolo, B., Cantalupo, G., Copioli, C., Fusco, C., Pelosi, A., . . Seri, S. (2012). Neonatal seizures and postneonatal epilepsy: a 7-y follow-up study. Pediatr Res, 72 (2), 186-193. doi:10.1038/pr.2012.66

Richards, S., Aziz, N., Bale, S., Bick, D., Das, S., Gastier-Foster, J., . . . Committee, A. L. Q. A. (2015). Standards and guidelines for the interpretation of sequence variants: a joint consensus recommendation of the American College of Medical Genetics and Genomics and the Association for Molecular Pathology. Genet Med, 17 (5), 405-424. doi:10.1038/gim.2015.30

Ronen GM, Buckley D, Penney S, \& Streiner DL. (2007). Long-term prognosis in children with neonatal seizures: a population based study. Neurology, 69 , 1816-1822.

Rothlisberger, B., Hoigne, I., Huber, A. R., Brunschwiler, W., \& Capone Mori, A. (2010). Deletion of 7q11.21-q11.23 and infantile spasms without deletion of MAGI2. Am J Med Genet A, 152A (2), 434-437. doi:10.1002/ajmg.a.33220 
Salzmann, A., Guipponi, M., Lyons, P. J., Fricker, L. D., Sapio, M., Lambercy, C., . . Malafosse, A. (2012). Carboxypeptidase A6 gene (CPA6) mutations in a recessive familial form of febrile seizures and temporal lobe epilepsy and in sporadic temporal lobe epilepsy. Hum Mutat, 33 (1), 124-135. doi:10.1002/humu.21613

Sapio, M. R., Vessaz, M., Thomas, P., Genton, P., Fricker, L. D., \& Salzmann, A. (2015). Novel carboxypeptidase A6 (CPA6) mutations identified in patients with juvenile myoclonic and generalized epilepsy. PLoS One, 10 (4), e0123180. doi:10.1371/journal.pone.0123180

Shellhaas, R. A., Chang, T., Wusthoff, C. J., Soul, J. S., Massey, S. L., Chu, C. J., . . Neonatal Seizure Registry Study, G. (2017). Treatment Duration After Acute Symptomatic Seizures in Neonates: A Multicenter Cohort Study. J Pediatr, 181 , 298-301 e291. doi:10.1016/j.jpeds.2016.10.039

Srivastava, S., McMillan, R., Willis, J., Clark, H., Chavan, V., Liang, C., . . Mukherjee, K. (2016). X-linked intellectual disability gene CASK regulates postnatal brain growth in a non-cell autonomous manner. Acta Neuropathol Commun, 4 , 30. doi:10.1186/s40478-016-0295-6

Tsuchida, T. N., Wusthoff, C. J., Shellhaas, R. A., Abend, N. S., Hahn, C. D., Sullivan, J. E., . . American Clinical Neurophysiology Society Critical Care Monitoring, C. (2013). American clinical neurophysiology society standardized EEG terminology and categorization for the description of continuous EEG monitoring in neonates: report of the American Clinical Neurophysiology Society critical care monitoring committee. $J$ Clin Neurophysiol, 30 (2), 161-173. doi:10.1097/WNP.0b013e3182872b24

UniProt, C. (2019). UniProt: a worldwide hub of protein knowledge. Nucleic Acids Res, 47 (D1), D506-D515. doi:10.1093/nar/gky1049

van Vliet, R., Breedveld, G., de Rijk-van Andel, J., Brilstra, E., Verbeek, N., Verschuuren-Bemelmans, C., ... Maat-Kievit, A. (2012). PRRT2 phenotypes and penetrance of paroxysmal kinesigenic dyskinesia and infantile convulsions.Neurology, 79 (8), 777-784. doi:10.1212/WNL.0b013e3182661fe3

Wang, J., Lin, Z. J., Liu, L., Xu, H. Q., Shi, Y. W., Yi, Y. H., . . Liao, W. P. (2017). Epilepsy-associated genes. Seizure, 44 , 11-20. doi:10.1016/j.seizure.2016.11.030

Wang, K. K., Yang, Z., Sarkis, G., Torres, I., \& Raghavan, V. (2017). Ubiquitin C-terminal hydrolase-L1 (UCH-L1) as a therapeutic and diagnostic target in neurodegeneration, neurotrauma and neuro-injuries. Expert Opin Ther Targets, 21 (6), 627-638. doi:10.1080/14728222.2017.1321635

Zheng, Q., Huang, T., Zhang, L., Zhou, Y., Luo, H., Xu, H., \& Wang, X. (2016). Dysregulation of Ubiquitin-Proteasome System in Neurodegenerative Diseases. Front Aging Neurosci, 8 , 303. doi:10.3389/fnagi.2016.00303

Figure legends

Figure 1: KEGG Orthology categorization of pathogenic variants on whole exome.

\section{Hosted file}

Numis-Table 1.docx available at https://authorea.com/users/333586/articles/459751-wholeexome-sequencing-with-targeted-gene-analysis-and-epilepsy-after-acute-symptomaticneonatal-seizures

\section{Hosted file}

Numis-Table 2.docx available at https://authorea.com/users/333586/articles/459751-wholeexome-sequencing-with-targeted-gene-analysis-and-epilepsy-after-acute-symptomaticneonatal-seizures

\section{Hosted file}


Numis-Table 3.docx available at https://authorea.com/users/333586/articles/459751-wholeexome-sequencing-with-targeted-gene-analysis-and-epilepsy-after-acute-symptomaticneonatal-seizures

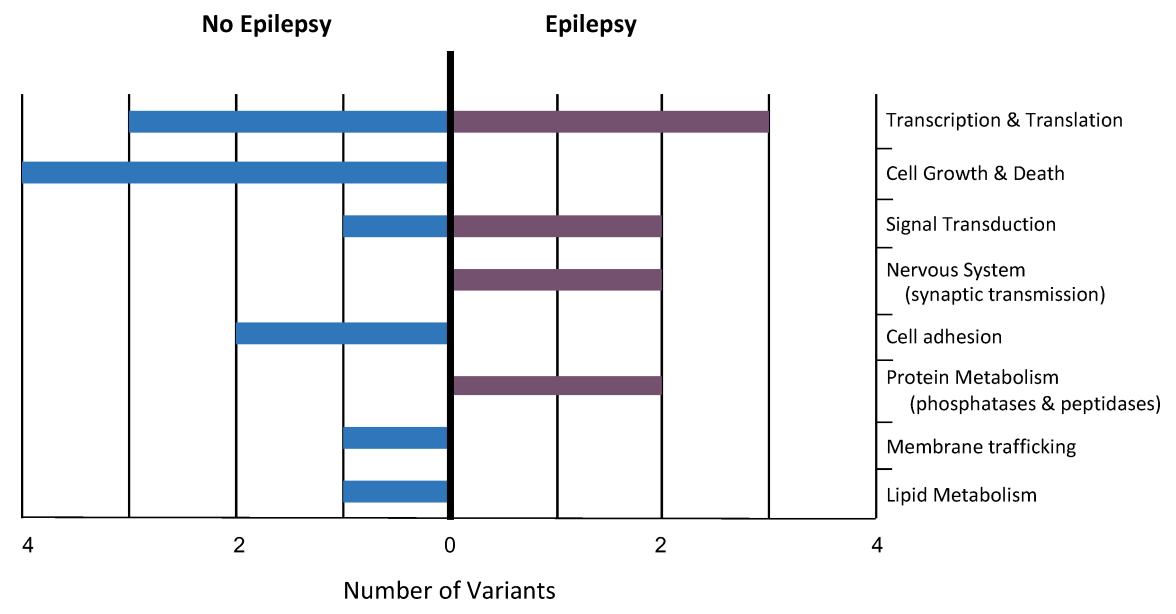

